\title{
A Better Characterization of Spinal Cord Damage in Multiple Sclerosis: A Diffusional Kurtosis Imaging Study
}

\author{
E. Raz, M. Bester, E.E. Sigmund, A. Tabesh, J.S. Babb, H. Jaggi, J. Helpern, R.J. Mitnick, and M. Inglese
}

\begin{abstract}
BACKGROUND AND PURPOSE: The spinal cord is a site of predilection for MS lesions. While diffusion tensor imaging is useful for the study of anisotropic systems such as WM tracts, it is of more limited utility in tissues with more isotropic microstructures (on the length scales studied with diffusion MR imaging) such as gray matter. In contrast, diffusional kurtosis imaging, which measures both Gaussian and non-Gaussian properties of water diffusion, provides more biomarkers of both anisotropic and isotropic structural changes. The aim of this study was to investigate the cervical spinal cord of patients with MS and to characterize lesional and normal-appearing gray matter and WM damage by using diffusional kurtosis imaging.
\end{abstract}

MATERIALS AND METHODS: Nineteen patients ( 13 women, mean age $=41.1 \pm 10.7$ years) and 16 controls $(7$ women, mean age $=35.6 \pm$ 11.2-years) underwent MR imaging of the cervical spinal cord on a 3T scanner (T2 TSE, T1 magnetization-prepared rapid acquisition of gradient echo, diffusional kurtosis imaging, T2 fast low-angle shot). Fractional anisotropy, mean diffusivity, and mean kurtosis were measured on the whole cord and in normal-appearing gray matter and WM.

RESULTS: Spinal cord T2-hyperintense lesions were identified in 18 patients. Whole spinal cord fractional anisotropy and mean kurtosis $(P=$ $.0009, P=.003)$, WM fractional anisotropy $(P=.01)$, and gray matter mean kurtosis $(P=.006)$ were significantly decreased, and whole spinal cord mean diffusivity $(P=.009)$ was increased in patients compared with controls. Mean spinal cord area was significantly lower in patients $(P=.04)$.

CONCLUSIONS: Diffusional kurtosis imaging of the spinal cord can provide a more comprehensive characterization of lesions and normal-appearing WM and gray matter damage in patients with MS. Diffusional kurtosis imaging can provide additional and complementary information to DTI on spinal cord pathology.

ABBREVIATIONS: $\mathrm{DKI}=$ diffusional kurtosis imaging; $\mathrm{EDSS}=$ Expanded Disability Status Scale; $\mathrm{GM}=$ gray matter; $\mathrm{MK}=$ mean kurtosis; $\mathrm{SC}=$ spinal cord

M ultiple sclerosis is a chronic inflammatory/demyelinating and neurodegenerative disease of the central nervous system and the most common cause of nontraumatic disability in

Received November 21, 2012; accepted after revision December 24.

From the Department of Radiology (E.R., M.B., E.E.S., A.T., J.S.B., H.J., R.J.M., M.I.), New York University School of Medicine, New York, New York; Department of Neurology and Psychiatry (E.R.), Sapienza University, Rome, Italy; Department of Neuroradiology (M.B.), Eppendorf-Hamburg University, Hamburg, Germany; Department of Neurology, Radiology and Neuroscience (M.I.), Mount Sinai School of Medicine, New York, New York; and Departments of Radiology and Radiological Science (A.T., J.H.) and Neurosciences (..H.), Medical University of South Carolina, Charleston, South Carolina.

E. Raz and M. Bester contributed equally to the article.

This work was supported in part by the National Institutes of Health $(\mathrm{NIH}$, grant R01NS051623).

Paper previously presented at: 19th Annual Meeting of the International Society for Magnetic Resonance in Medicine, June 4-9, 2011; Seattle, Washington.

Please address correspondence to Matilde Inglese, MD, Department of Neurology, Mount Sinai School of Medicine, One Gustave L. Levy Place, Box 1137. New York, NY 10029; e-mail: matilde.inglese@mssm.edu

-- Indicates open access to non-subscribers at www.ajnr.org

http://dx.doi.org/10.3174/ajnr.A3512 young adults. ${ }^{1}$ The spinal cord is a site of predilection for MS lesions, ${ }^{2}$ and SC damage contributes to the onset of neurologic deficits and the development of clinical disability. ${ }^{3}$ At postmortem examination, $86 \%$ of patients with MS have spinal cord lesions, ${ }^{2}$ and MR imaging cord abnormalities are detected in up to $89 \%$ of patients with established disease. ${ }^{4}$ However, previous MR imaging studies of patients with MS found a poor correlation between the number and volume of T2-hyperintense SC lesions and disability, ${ }^{5}$ very likely due to the presence of additional microscopic abnormalities in the so-called normal-appearing white matter, not detectable by conventional MR imaging techniques. ${ }^{6,7}$

Several pathologic studies of MS spinal cord samples have shown that not only SC WM but also GM is affected by demyelination and neuronal loss. ${ }^{8-11}$ Despite these reports and unlike findings reported in the brain, only a few in vivo MR imaging studies to date have investigated the presence and extent of spinal cord GM damage in patients with MS. ${ }^{12-14}$ 
DTI is a clinically important MR imaging method used to measure the micron scale displacement of water molecules in the brain and can provide information about tissue structural integrity at a microscopic level. ${ }^{15}$ Recent DTI studies of the spinal cord in patients with MS indeed showed a significant decrease in fractional anisotropy, a measure of WM fiber integrity and directionality, compared with healthy controls in both lesions and normalappearing tissue. ${ }^{16-24}$ The DTI model, however, is based on the assumption that the displacement probability function of diffusing water molecules follows a Gaussian distribution, which is only the first approximation to the true behavior in complex tissue. Therefore, while the anisotropic sensitivity of DTI is useful for the study of highly organized systems such as WM tracts, it provides fewer useful biomarkers in tissues such as GM. This is because for the length scales studied with diffusion MR imaging (ie, a few microns), the microstructure of GM is characterized as isotropic. Conversely, non-Gaussian diffusion may be more sensitive to subtle pathologic changes in GM because diffusional non-Gaussianity is a direct consequence of diffusion barriers and compartments, regardless of the presence of microstructural anisotropy. ${ }^{25}$

Among the MR imaging techniques that have been developed to assess non-Gaussian diffusion in spinal cord tissue, ${ }^{13,15}$ diffusional kurtosis imaging ${ }^{25}$ represents the minimal extension of DTI that, in acquisition times compatible with clinical application, enables the quantification of non-Gaussian diffusion through the estimation of the diffusional kurtosis. A DKI-derived metric, the mean kurtosis, provides a measure of the deviation of the diffusion probability distribution from a Gaussian form. ${ }^{25,26}$ MK has been shown to be sensitive to structural changes in both anisotropic tissue, such as WM, and isotropic tissue such as GM and therefore may provide information on tissue microarchitecture complementary to that given by fractional anisotropy and mean diffusivity. ${ }^{25}$ Using a moderately expanded diffusion-sampling scheme, MK can be obtained simultaneously with conventional DTI, ${ }^{27,28}$ and it has already been used to study the brain in normal aging, ${ }^{27}$ Alzheimer disease, ${ }^{29}$ traumatic brain injury, ${ }^{30}$ brain tumors, ${ }^{31}$ stroke, ${ }^{32}$ and the spinal cord in patients with cervical myelopathy. ${ }^{33}$

The aims of our study were the following: 1) to investigate global and regional (WM and GM) microstructural abnormalities in the cervical spinal cord of patients with MS compared with healthy controls by using MK, mean diffusivity, and fractional anisotropy; 2) to determine whether cervical cord MK provides additional information about the underlying structural abnormalities compared with mean diffusivity and fractional anisotropy; 3 ) to assess the relationship between cervical spinal cord DKI-derived parameters and measures of cord area and volume; and 4) to determine the clinical impact of global and regional cervical spinal cord DKI-derived metrics.

\section{MATERIALS AND METHODS \\ Patients}

The study was approved by the institutional review board of New York University and complies with the Health Insurance Portability and Accountability Act. Written informed consent was obtained from all participants. For this prospective study, we recruited 21 consecutive patients with clinically definite $\mathrm{MS}^{34}$ who were referred from the New York University MS Center to our

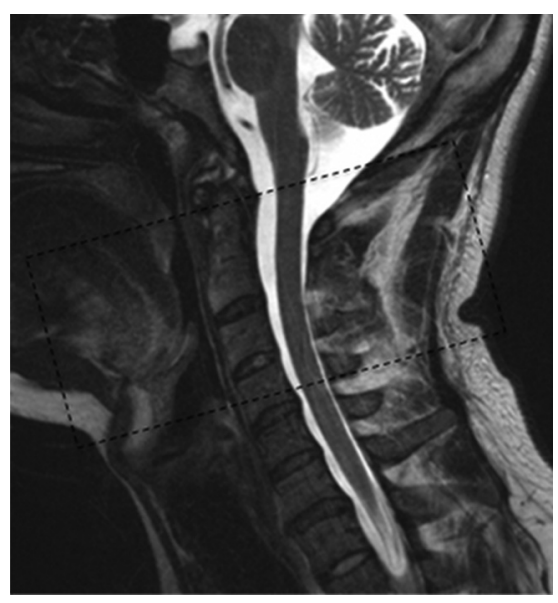

FIG 1. Sagittal T2 image from a healthy control demonstrating the coverage of the axial diffusion kurtosis sequence, centered on the C2-3 intervertebral space.

department for cervical spinal cord MR imaging between August 2009 and March 2010 and who met the following inclusion criteria: 1) had a diagnosis of relapsing-remitting MS, 2) were between 18 and 60 years of age, and 3) had an absence of clinical relapse and/or steroid treatment in the 3 months before MR imaging. The exclusion criteria were the presence of other relevant diseases and contraindications to performing MR imaging. Two patients were excluded because of claustrophobia.

All subjects underwent a neurologic evaluation. The following demographic and clinical variables were collected for each patient: age, disease duration from diagnosis, disease duration from symptom onset, and level of disability assessed by the EDSS. The 19 patients had a mean age of $41.1 \pm 10.7$ years. There were 13 women (mean age $=40.1 \pm 10.7$ years) and 6 men (mean age $=$ $43.1 \pm 11.5$ years $)$.

Sixteen healthy controls (mean age $=35.6 \pm 11.2$ years, 9 men and 7 women) with no known brain abnormalities and no neurologic symptoms were recruited as controls and given the same MR imaging examination as described above.

\section{MR Imaging Acquisition}

MR imaging was performed by using a 3T scanner (Tim Trio; Siemens, Erlangen, Germany) with a 4-channel neck phased array coil, and the following imaging protocol was used in all subjects during a single MR imaging session:

1) A sagittal T2-weighted TSE sequence with 15 contiguous sections $(\mathrm{TR}=3500 \mathrm{~ms}, \mathrm{TE}=113 \mathrm{~ms}, \mathrm{FOV}=240 \mathrm{~mm}$, matrix $=$ $512 \times 512$, section thickness $=3 \mathrm{~mm}$, in-plane resolution $=$ $\left.0.46 \times 0.46 \mathrm{~mm}^{2}\right)$.

2) Sagittal T1-weighted 3D MPRAGE on 24 contiguous sections $(\mathrm{TR}=2300 \mathrm{~ms}, \mathrm{TE}=2.98 \mathrm{~ms}, \mathrm{TI}=900 \mathrm{~ms}, \mathrm{FOV}=240$ $\mathrm{mm}$, matrix $=230 \times 256$, isotropic voxel size $\left.=1 \times 1 \times 1 \mathrm{~mm}^{3}\right)$.

3) An axial DKI sequence, centered on the spinal intervertebral disk between C2 and C3 (a slab position is visible in Fig 1), by using a twice-refocused spin-echo diffusion sequence with an echo-planar imaging readout ${ }^{35}$ with 30 different diffusion-encoding directions by using an optimized sampling strategy ${ }^{36,37}$ and 20 contiguous axial sections. For each direction, $6 \mathrm{~b}$-values $(0$, $\left.500,1000,1500,2000,2500 \mathrm{~s} / \mathrm{mm}^{2}\right)$ were used $(\mathrm{TR}=3100 \mathrm{~ms}$, 

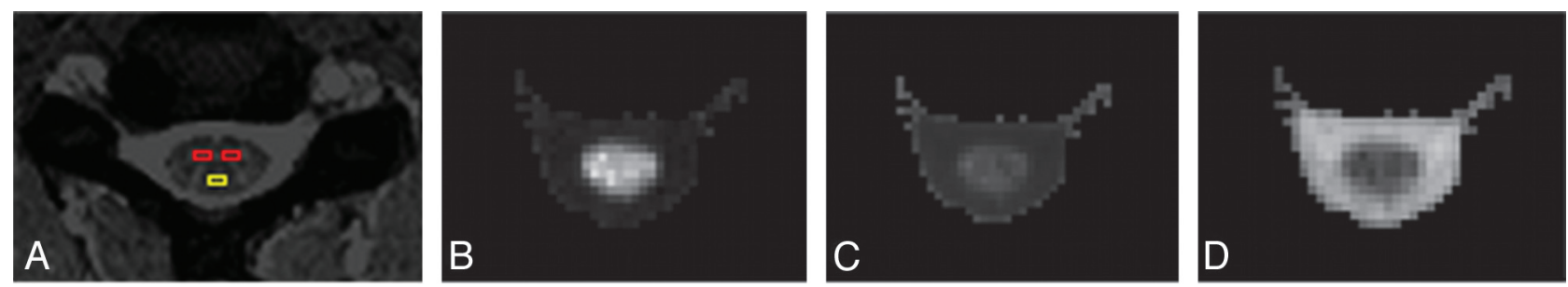

FIG 2. Axial T2 image (A) of the cervical spinal cord at $C 2$ shows the location of ROls placed in the gray matter of the ventral horns (red) and white matter of the dorsal column (yellow). Corresponding fractional anisotropy (B), mean kurtosis (C), and mean diffusivity $(D)$ maps. The size of the region of interest is 2 voxels $\left(3.1 \mathrm{~mm}^{2}\right)$. Diffusion and kurtosis tensors were calculated by using in-house software to generate maps of the derived parameters, fractional anisotropy, mean diffusivity, and MK.

$\mathrm{TE}=110 \mathrm{~ms}, \mathrm{FOV}=160 \mathrm{~mm}$, matrix $=128 \times 128$, section thickness $=3 \mathrm{~mm}$, number of averages $=2$, in-plane resolution $=$ $1.25 \times 1.25 \mathrm{~mm}^{2}$, acquisition time $=15$ minutes 7 seconds).

4) An axial T2-weighted fast low-angle shot MR imaging sequence, centered on the spinal intervertebral disk between $\mathrm{C} 2$ and $\mathrm{C} 3$, with 24 contiguous sections $(\mathrm{TR}=500 \mathrm{~ms}$, TE $=7.38 \mathrm{~ms}$, $\mathrm{FOV}=176 \mathrm{~mm}$, matrix $=192 \times 320$, section thickness $=3 \mathrm{~mm}$, in-plane resolution $=0.68 \times 0.68 \mathrm{~mm}^{2}$ ).

5) Only in the 19 patients, an additional sagittal T2 STIR sequence with 13 contiguous sections was acquired ( $\mathrm{TR}=4500 \mathrm{~ms}$, $\mathrm{TE}=53 \mathrm{~ms}, \mathrm{TI}=200 \mathrm{~ms}, \mathrm{FOV}=240 \mathrm{~mm}$, matrix $=480 \times 480$, section thickness $=3 \mathrm{~mm}$, in-plane resolution $=0.37 \times 0.37$ $\left.\mathrm{mm}^{2}\right)$.

\section{Postprocessing}

Image data processing and evaluation were performed by an MR imaging expert and a neuroradiologist. T2-weighted and STIR images were used to identify and count the lesions in the spinal cord.

\section{DKI/DTI Analysis}

Diffusion and kurtosis tensors were calculated by using in-house software (Diffusion and Kurtosis Estimator) in Matlab, Version 7.0 (MathWorks, Natick, Massachusetts); maps of derived parameters, fractional anisotropy, mean diffusivity, and MK, were generated after first coregistering all diffusion-weighted images with a $2 \mathrm{D}$ rigid-body algorithm. ${ }^{38}$ After we derived apparent diffusion and kurtosis along each applied gradient direction via a quadratic fit to the logarithm of the signal intensity, the analysis algorithm, described in detail in Tabesh et al, ${ }^{38}$ used a linearly constrained linear least-squares formulation to solve for both the diffusion and kurtosis tensors at each voxel. Using region-of-interest analysis, we measured mean fractional anisotropy, mean diffusivity, and MK values of the entire cross-sectional cord area between $\mathrm{C} 1$ and C4 (5 contiguous sections for each level), generating 20 values for each of the DTI metrics in each subject (altogether 380 SC sections for 19 patients). Of 380 sections, 165 included T2 visible lesions, whereas the remaining 215 were classified as normal-appearing spine tissue.

The lesions were outlined on the T2-weighted images by using Medical Image Processing, Analysis, and Visualization (http:// mipav.cit.nih.gov). Then, the outlined regions of interest were automatically transferred onto the corresponding DKI/DTI maps, and the mean lesional fractional anisotropy, mean diffusivity, and MK values were measured. In addition, mean fractional anisotropy, mean diffusivity, and MK values of normal-appearing
GM and WM were measured by using rectangular ROIs (size: 2 voxels, $3.1 \mathrm{~mm}^{2}$ ) placed on the T2 images in the left and right ventral horns and in the dorsal columns over 2 contiguous sections at the $\mathrm{C} 2$ level, as described in Freund et al (Fig 2). ${ }^{39}$ The regions of interest were then opened on the registered DKI/DTI maps, and the mean values for GM/WM were calculated by averaging the results obtained in all ROIs (4 ROIs for GM, 2 ROIs for WM). This regional analysis was performed on patients (14 of 19) who did not show any lesions at the $\mathrm{C} 2$ level.

\section{Cervical Spinal Cord Area and Volume Measurement}

Cervical cord area and volume were measured on T1 MPRAGE images. For each subject, five 3-mm-thick axial sections perpendicular to the spinal cord were reconstructed, centering on the C2-3 intervertebral disk, and an established semiautomated technique was used to measure the cross-sectional cord area. ${ }^{40}$ The volume of the cervical cord section between C2 and C4 was evaluated by using BrainMask software (http://www.nitrc.org/ projects/brainmask $)^{41}$ by using $3 \mathrm{D}$ edge-detection algorithms, allowing volume selection with 3 simultaneous reference planes. Because the software is suboptimal for the evaluation of a curved object, the volume between C2 and C4 was selected as representative of the entire cervical cord volume.

\section{Statistical Analysis}

Statistical analysis was performed by using SAS (SAS Institute, Cary, North Carolina). The DKI/DTI values were represented for each subject as an average over the values recorded for each spine location. As a result, each subject contributed 1 value of each of MK, mean diffusivity, and fractional anisotropy for the total SC (including lesions) and over the normal-appearing SC. Additionally, in patients with MS without visible lesions at the $\mathrm{C} 2$ level and in healthy controls, fractional anisotropy, mean diffusivity, and MK were measured in normal-appearing spinal cord tissue (GM and WM). Analysis of covariance was used to compare patients and controls in terms of the total and regional values of MK, mean diffusivity, and fractional anisotropy, adjusting for age, sex, and disease duration, and to compare the 2 groups of patients stratified according to the EDSS score (group 1: EDSS score $\leq 2.5$, female/male ratio $=9: 2$, mean age $=39.0 \pm 8.9$ years, mean disease duration $=8.5 \pm 6.0$ years; group 2: EDSS score $\geq 3.0$, female $/$ male ratio $=4: 4$, mean age $=44.0 \pm 12.0$ years, mean disease duration $=5.7 \pm 3.0$ years $)$ adjusting for age, sex, disease duration, number of lesions, and cord area. 

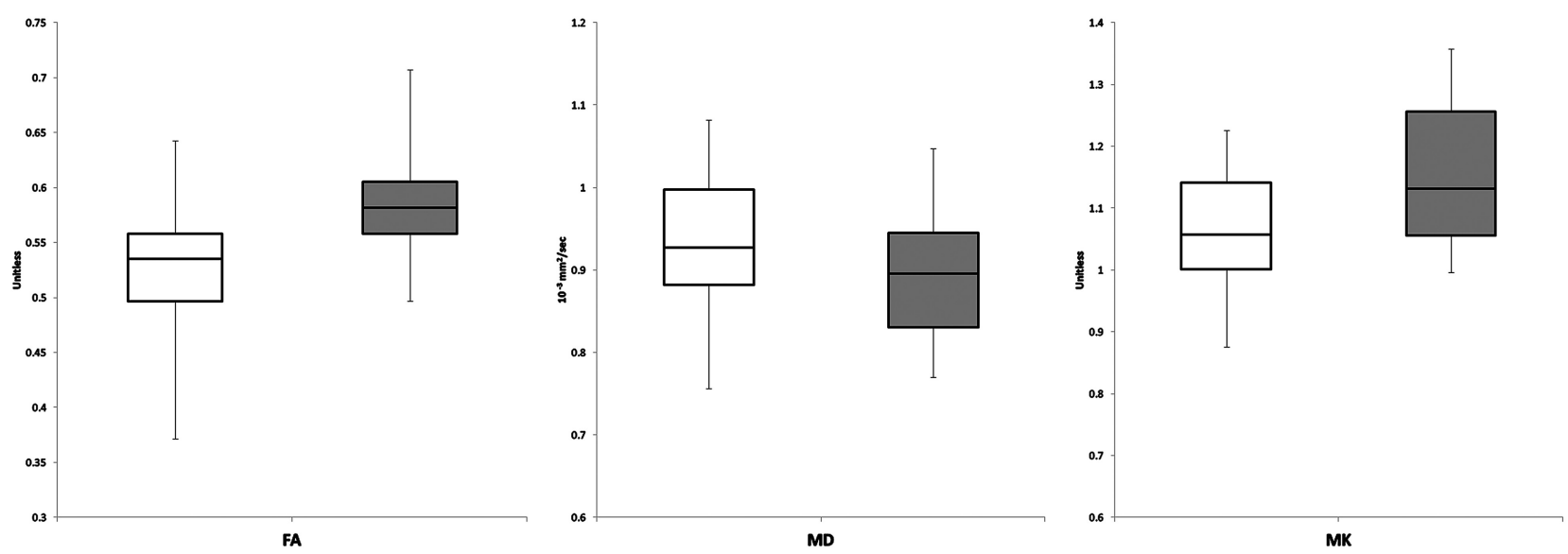

FIG 3. Box-and-whisker plots show the distribution of fractional anisotropy, mean diffusivity, and MK values in patients (white boxes) and in healthy controls (gray boxes). ANCOVA comparing patients with controls and adjusted for age, sex, and disease duration gave the following $P$ values: fractional anisotropy, $P=.0009$; mean diffusivity, $P=.009 ; \mathrm{MK}, P=.003$.

Table 1: The mean of DKI/DTI measures of the whole spinal cord over sections with lesions $(n=145)$ and over sections of normal-appearing spine tissue $(n=215)$ in patients with MS $^{\mathrm{a}}$

\begin{tabular}{lccc}
\hline Measure $^{c}$ & Lesions & NAST & $\boldsymbol{P}_{\text {Value }}$ \\
\hline FA & $0.42 \pm 0.02$ & $0.52 \pm 0.04$ & $<.0001$ \\
MD & $1.17 \pm 0.08$ & $0.92 \pm 0.05$ & $<.0001$ \\
MK & $0.91 \pm 0.03$ & $1.08 \pm 0.08$ & $<.0001$ \\
\hline
\end{tabular}

Note:-NAST indicates normal-appearing spine tissue; FA, fractional anisotropy; MD, mean diffusivity.

a Only data from subjects with lesions $(n=18)$ were included in the computations.

${ }^{\mathrm{b}} \mathrm{P}$ values are from ANCOVA, comparing lesions to normal-appearing spine tissue in terms of the indicated DKI measure adjusted for age, sex, and disease duration.

${ }^{c} \mathrm{FA}$ and MK are unitless. MD is measured in $\times 10^{-3} \mathrm{~mm}^{2} / \mathrm{s}$

Table 2: The mean of DKI measures at the C2-3 level in patients with MS and controls

\begin{tabular}{lcccc}
\hline Measure & Location & Controls & Patients with MS & $\boldsymbol{P}_{\text {Value }}$ \\
\hline FA & GM & $0.81 \pm 0.11$ & $0.70 \pm 0.19$ & .4 \\
& WM & $0.63 \pm 0.07$ & $0.52 \pm 0.07$ & .01 \\
MD & GM & $0.56 \pm 0.08$ & $0.72 \pm 0.21$ & .1 \\
& WM & $0.86 \pm 0.14$ & $0.99 \pm 0.13$ & .06 \\
MK & GM & $1.16 \pm 0.13$ & $1.11 \pm 0.09$ & .006 \\
& WM & $1.15 \pm 0.17$ & $1.08 \pm 0.12$ & .08 \\
\hline
\end{tabular}

Note:-FA indicates fractional anisotropy; MD, mean diffusivity.

a $P$ values are from ANCOVA, comparing patients with normal-appearing spine tissue at the $C 2-3$ level $(n=14)$ with controls in terms of WM and GM DKI measures adjusted for age, sex, and disease duration.

The error variance was allowed to differ across subject groups to remove the unnecessary assumption-of-variance homogeneity. A paired-sample $t$ test was used to compare WM and GM at the lesion level with corresponding normal-appearing tissue in terms of each DKI measure.

Pearson correlations were used to characterize the association of the DKI measures from the whole cervical spine and from the GM and WM, separately, with spinal cord volume and area and EDSS. All $P$ values are reported as 2-sided significance levels without adjustment for multiple comparisons and were considered statistically significant when $P<.05$.

\section{RESULTS}

Nineteen patients had a median EDSS score of 2.5 (range $=1-6.5$ ). Mean disease duration from the diagnosis was $7.3 \pm 5.2$ years, while mean disease duration from symptom onset was $10.1 \pm 7.8$ years. Fifty-nine T2 hyperintense lesions were identified in 18 of 19 patients, with a median of 3 lesions per patient (range $=0-8$ ).
Cross-sectional mean MK and fractional anisotropy (both unitless) and mean diffusivity $\left(\times 10^{-3} \mathrm{~mm}^{2} / \mathrm{s}\right)$ values in patients and controls for the whole cervical spinal cord examined are reported in Fig 3. Compared with controls, fractional anisotropy and MK were significantly decreased (respectively, $P=.0009$ and $P=.003)$ and mean diffusivity was increased $(P=.009)$ in patients.

In patients with MS, when mean diffusivity, MK, and fractional anisotropy values of sections with lesions were compared with the values in sections of normal-appearing spine tissue, the 3 parameters differed significantly (Table 1): fractional anisotropy and MK were lower and mean diffusivity was higher in lesions than in normal-appearing spine tissue (all $P<.0001)$.

Mean MK, fractional anisotropy, and mean diffusivity values of GM and WM ROIs were compared at the C2-3 level in healthy subjects and patients with MS without visible lesions at that level $(n=14)$; the results are reported in Table 2. In patients, normalappearing WM fractional anisotropy and normal-appearing GM MK values were significantly decreased compared with those in healthy controls while mean diffusivity, GM fractional anisotropy, and WM MK were not significantly different across the 2 groups of subjects.

MK and mean diffusivity values were significantly inversely correlated to each other in the whole SC, in lesions, and in normal $\mathrm{WM}(r=-0.76, P=.0006 ; r=-0.2, P=.01$; and $r=-0.69, P=$ .001 , respectively) but not in the gray matter $(P=.9)$.

The mean cervical cord cross-sectional area measured at C2-3 was $0.93 \pm 0.06 \mathrm{~cm}^{2}$ in the patient group, significantly lower than that in the control group $\left(0.94 \pm 0.08 \mathrm{~cm}^{2}, P=.04\right)$. Mean C2-4 volume of the cervical spinal cord was $2.70 \pm 0.34 \mathrm{~cm}^{3}$ in patients and $2.96 \pm 0.36 \mathrm{~cm}^{3}$ in controls $(P=.7)$. 
Table 3: The mean of DKI measures for the 2 groups, based on the median EDSS score ${ }^{a}$

\begin{tabular}{ccccc}
\hline Measure & Location & Group 1 $(\boldsymbol{n}=11)$ & Group 2 $(\boldsymbol{n}=\mathbf{8})$ & $\boldsymbol{P}$ Value \\
\hline FA & WC & $0.54 \pm 0.04$ & $0.49 \pm 0.06$ & .002 \\
& GM & $0.74 \pm 0.19$ & $0.65 \pm 0.22$ & .01 \\
\multirow{3}{*}{ MD } & WM & $0.53 \pm 0.09$ & $0.50 \pm 0.09$ & .1 \\
& WC & $0.92 \pm 0.09$ & $0.95 \pm 0.09$ & .001 \\
& GM & $0.70 \pm 0.21$ & $0.75 \pm 0.23$ & .001 \\
MK & WM & $0.96 \pm 0.15$ & $1.03 \pm 0.14$ & .5 \\
& WC & $1.07 \pm 0.11$ & $1.06 \pm 0.10$ & .01 \\
& GM & $1.13 \pm 0.13$ & $1.10 \pm 0.09$ & $<.0001$ \\
& WM & $1.09 \pm 0.15$ & $1.08 \pm 0.14$ & .3 \\
\hline
\end{tabular}

Note:-FA indicates fractional anisotropy; MD, mean diffusivity; WC, whole cord.

${ }^{a}$ Group 1: EDSS score $\leq 2.5$; group 2: EDSS score $\geq 3.0$.

${ }^{\mathrm{b}} \mathrm{P}$ values are from ANCOVA, comparing groups 1 and 2 in terms of the indicated DKI measure adjusted for age, sex, and disease duration.

Finally, we did not find any significant correlation of SC, MK, fractional anisotropy, and mean diffusivity values with either spine volume or spine area, except for the WM MK and cervical spine volume ( $r=0.58$ and $P=.0089)$.

Likewise, no significant correlations were found among any MK, fractional anisotropy, and mean diffusivity values and EDSS. However, when the patients were stratified into 2 groups on the basis of the median EDSS score, more disabled patients (group 2) showed significantly lower fractional anisotropy and MK and higher mean diffusivity of the total cervical SC after correcting for age, sex, disease duration, and number of lesions. Most interesting, the locally sampled GM region showed lower MK and fractional anisotropy and higher mean diffusivity in the more disabled class, while the locally sampled WM did not show significant differences between the EDSS groups. Total and regional SC MK, fractional anisotropy, and mean diffusivity values from the 2 groups of patients are compiled in Table 3.

\section{DISCUSSION}

The spinal cord is frequently involved in MS, and it is a clinically eloquent site of damage with a relevant contribution to the accumulation of irreversible physical disability. ${ }^{42}$ There is growing evidence indicating that MS tissue damage in both the brain and SC is not limited to WM but also involves the GM. ${ }^{13}$ Our DKI study in patients with MS showed extensive cervical spinal cord damage and particularly more severely at the lesional level than in normal-appearing tissue. This is in agreement with previous DTI studies that found decreased fractional anisotropy values and increased mean diffusivity values in the cervical SC of patients with MS in comparison with healthy controls. ${ }^{16,20}$ The pathologic substrate underlying the changes in DTI metrics is represented by demyelination and axonal loss occurring in spinal cord T2-visible lesions and in normal-appearing tissue. ${ }^{43,44}$ Both demyelination and axonal loss lead to an increase of the extracellular volume, ${ }^{8}$ which is reflected by reduced fractional anisotropy and increased mean diffusivity. In addition, intracellular abnormalities that determine the formation of new barriers, such as cell debris, inflammatory changes, and gliosis, restrict water isotropically and can cause reductions in fractional anisotropy. ${ }^{8}$

More recently, several pathologic studies ${ }^{8-11,45}$ demonstrated that extensive demyelination and neuronal loss occur in the SC GM. Although neuronal loss appears to be predominantly related to local GM plaques, interneuron atrophy occurs in both myelinated and demyelinated areas. ${ }^{46}$ In line with these findings, our study showed a significant decrease of MK in the SC normal-appearing GM of patients compared with controls. Although we can only speculate about the pathologic nature of the GM MK change, it is likely to reflect both the presence of GM lesions below the resolution of conventional T2-weighted scans and the degeneration of GM neurons secondary to axonal transactions in the WM. Although we did not find a significant association between SC lesion count and GM MK ( $r=0.3, P=.1)$,

there was a trend toward a significant positive association between the decrease of WM MK and that of GM MK $(r=0.44, P=.06)$, suggesting a possible interplay between injury in WM and GM.

While in WM, fractional anisotropy was sensitive to diseaserelated tissue damage and there was a trend toward significance for mean diffusivity and MK, in GM, only MK was significantly different between patients and controls, suggesting that it could be a more sensitive metric for evaluating the GM than mean diffusivity and fractional anisotropy. While standard DTI metrics can characterize the diffusion of water molecules in $3 \mathrm{D}$ space, they are not able to provide more specific information about its microenvironment. For example, mean diffusivity can indicate that the average water diffusion distance is small, but it cannot differentiate whether the small diffusion distance is due to a more viscous environment or the presence of more barriers, such as cell membranes. ${ }^{25}$ A decrease in MK more clearly represents a reduction in diffusional heterogeneity; in the case of spinal cord tissue, this is very likely associated with degenerative changes and neuronal shrinkage. ${ }^{25,26,29,47}$ Hence, MK is a more natural indicator of tissue microstructural properties because diffusional nonGaussianity is a direct consequence of the presence of barriers and compartments.

The lack of correlation between average mean diffusivity and MK in the GM of our patients and the poor correlation between the 2 metrics in lesional SC further supports the notion that MK of the GM can provide information about the underlying tissue microarchitecture that is different and complementary to that obtained with conventional DTI.

We did not find a significant correlation between any of the DKI-derived metrics in the global and regional cervical SC and the EDSS score. This result may be influenced by the small sample size and the narrow range of EDSS scores for most patients. However, when the patients were stratified on the basis of the median EDSS score into 2 groups with high and low disability, more disabled patients showed significant differences in total SC and GM DKI metrics compared with less disabled patients. This finding suggests that the severity of SC GM damage might have a role in the development of irreversible disability and is consistent with the results of previous MR imaging studies of SC GM by using magnetization-transfer imaging. ${ }^{12,14}$

This study has some limitations. The number of patients enrolled allowed us to investigate the potential of a new MR imaging technique such as DKI, but it is relatively low, and our results will 
need to be confirmed in a larger sample including patients with different disease courses.

The small size of the SC and the moderate resolution of DWI sequences make it difficult to avoid partial volume effects especially in relation to GM and WM assessment; however, the group level changes in the DKI metrics are robust and consistent with expectations. We acknowledge that even though patients and controls were not exactly sex-matched, all our statistical analyses were corrected for age and sex, thus limiting the possibility of lack of sex-matching being a confounding factor. In addition, although we found a moderate correlation between WM injury and cord volume as already reported in the literature, ${ }^{9}$ we found no association between any of the GM DKI metrics and cord volume or area, suggesting that the difference between patients and controls is not entirely attributable to partial volume effects. Furthermore, because the spatial resolution of our DKI images did not allow a more detailed identification of the ventral and lateral white matter columns, we limited our laminar assessment to the dorsal columns and the central GM region. Finally, a better interpretation of MK changes in the GM of the MS spinal cord will be available when the pathologic substrates are identified by postmortem high-resolution MR imaging and/or quantitative histopathologic studies.

\section{CONCLUSIONS}

DKI of the cervical spinal cord in patients with MS can be useful for a better characterization of lesions and normal-appearing white and gray matter damage, with acquisition times compatible with clinical applications. With DKI, MK can be obtained simultaneously with conventional DTI-derived parameters; because its structural sensitivity is not limited to spatially oriented tissue structures, it can provide additional information complementary to that provided by DTI. Although our SC DKI findings are preliminary, our study supports the results of previous studies on DTI of the SC and suggests that gray matter damage may play a key role in accrual of disability in MS.

\section{ACKNOWLEDGMENTS}

E.R. thanks Centro Interuniversitario di Formazione Internazionale (H2CU) from Sapienza University of Rome for its support.

Disclosures: Eric E. Sigmund-RELATED: Support for Travel to Meetings for the Study or Other Purposes: American Society of Spine Radiology, Comments: invited to give educational lecture on ultra-high-field spinal cord MRI, with travel expenses compensated at the Annual Symposium of the American Society of Spine Radiology, February 16-19, 2012. Matilde Inglese-RELATED: Grant: National Institutes of Health $(\mathrm{NIH}){ }^{*}$ *Money paid to the institution.

\section{REFERENCES}

1. Noseworthy JH, Lucchinetti CF, Rodriguez M, et al. Multiple sclerosis. N Engl J Med 2000;343:938-52

2. Ikuta F, Zimmerman HM. Distribution of plaques in seventy autopsy cases of multiple sclerosis in the United States. Neurology 1976;26:26-28

3. Bjartmar C, Wujek JR, Trapp BD. Axonal loss in the pathology of MS: consequences for understanding the progressive phase of the disease. J Neurol Sci 2003;206:165-71

4. Rocca MA, Mastronardo G, Horsfield MA, et al. Comparison of three MR sequences for the detection of cervical cord lesions in patients with multiple sclerosis. AJNR Am J Neuroradiol 1999;20:1710-16
5. Kidd D, Thorpe JW, Thompson AJ, et al. Spinal cord MRI using multi-array coils and fast spin echo. II. Findings in multiple sclerosis. Neurology 1993;43:2632-37

6. Barkhof F. The clinico-radiological paradox in multiple sclerosis revisited. Curr Opin Neurol 2002;15:239-45

7. Filippi M, Campi A, Colombo B, et al. A spinal cord MRI study of benign and secondary progressive multiple sclerosis. J Neurol 1996;243:502-05

8. Nijeholt GJ, Bergers E, Kamphorst W, et al. Post-mortem high-resolution MRI of the spinal cord in multiple sclerosis: a correlative study with conventional MRI, histopathology and clinical phenotype. Brain 2001;124:154-66

9. Gilmore CP, DeLuca GC, Bö L, et al. Spinal cord atrophy in multiple sclerosis caused by white matter volume loss. Arch Neurol 2005;62:1859-62

10. Gilmore CP, Bö L, Owens T, et al. Spinal cord gray matter demyelination in multiple sclerosis-a novel pattern of residual plaque morphology. Brain Pathol 2006;16:202-08

11. Gilmore CP, Geurts JJ, Evangelou N, et al. Spinal cord grey matter lesions in multiple sclerosis detected by post-mortem high field MR imaging. Mult Scler 2009;15:180-88

12. Agosta F, Absinta M, Sormani MP, et al. In vivo assessment of cervical cord damage in MS patients: a longitudinal diffusion tensor MRI study. Brain 2007;130:2211-19

13. Farrell JA, Smith SA, Gordon-Lipkin EM, et al. High b-value q-space diffusion-weighted MRI of the human cervical spinal cord in vivo: feasibility and application to multiple sclerosis. Magn Reson Med 2008;59:1079-89

14. Zackowski KM, Smith SA, Reich DS, et al. Sensorimotor dysfunction in multiple sclerosis and column-specific magnetization transfer-imaging abnormalities in the spinal cord. Brain 2009; 132:1200-09

15. Inglese $M$, Bester M. Diffusion imaging in multiple sclerosis: research and clinical implications. NMR Biomed 2010;23:865-72

16. Agosta F, Benedetti B, Rocca MA, et al. Quantification of cervical cord pathology in primary progressive MS using diffusion tensor MRI. Neurology 2005;64:631-35

17. Benedetti B, Valsasina P, Judica E, et al. Grading cervical cord damage in neuromyelitis optica and MS by diffusion tensor MRI. Neurology 2006;67:161-63

18. Ciccarelli O, Wheeler-Kingshott CA, McLean MA, et al. Spinal cord spectroscopy and diffusion-based tractography to assess acute disability in multiple sclerosis. Brain 2007;130:2220-31

19. Clark CA, Werring DJ, Miller DH. Diffusion imaging of the spinal cord in vivo: estimation of the principal diffusivities and application to multiple sclerosis. Magn Reson Med 2000;43:133-38

20. Hesseltine SM, Law M, Babb J, et al. Diffusion tensor imaging in multiple sclerosis: assessment of regional differences in the axial plane within normal-appearing cervical spinal cord. AJNR Am J Neuroradiol 2006;27:1189-93

21. Ohgiya Y, Oka M, Hiwatashi A, et al. Diffusion tensor MR imaging of the cervical spinal cord in patients with multiple sclerosis. Eur Radiol 2007; 17:2499-504

22. Reich DS, Smith SA, Zackowski KM, et al. Multiparametric magnetic resonance imaging analysis of the corticospinal tract in multiple sclerosis. Neuroimage 2007;38:271-79

23. Valsasina P, Rocca MA, Agosta F, et al. Mean diffusivity and fractional anisotropy histogram analysis of the cervical cord in MS patients. Neuroimage 2005;26:822-28

24. van Hecke W, Nagels G, Emonds G, et al. A diffusion tensor imaging group study of the spinal cord in multiple sclerosis patients with and without T2 spinal cord lesions. J Magn Reson Imaging 2009;30:25-34

25. Jensen JH, Helpern JA. MRI quantification of non-Gaussian water diffusion by kurtosis analysis. NMR Biomed 2010;23:698-710

26. Wu EX, Cheung MM. MR diffusion kurtosis imaging for neural tissue characterization. NMR Biomed 2010;23:836-48 
27. Falangola MF, Jensen JH, Babb JS, et al. Age-related non-Gaussian diffusion patterns in the prefrontal brain. J Magn Reson Imaging 2008;28:1345-50

28. Hui ES, Cheung MM, Qi L, et al. Towards better MR characterization of neural tissues using directional diffusion kurtosis analysis. Neuroimage 2008;42:122-34

29. Lu H, Jensen JH, Ramani A, et al. Three-dimensional characterization of non-gaussian water diffusion in humans using diffusion kurtosis imaging. NMR Biomed 2006;19:236-47

30. Grossman EJ, Ge Y, Jensen JH, et al. Thalamus and cognitive impairment in mild traumatic brain injury: a diffusional kurtosis imaging study. J Neurotrauma 2012;29:2318-27

31. Raab P, Hattingen E, Franz K, et al. Cerebral gliomas: diffusional kurtosis imaging analysis of microstructural differences. Radiology 2010;254:876-81

32. Jensen JH, Falangola MF, Hu C, et al. Preliminary observations of increased diffusional kurtosis in human brain following recent cerebral infarction. NMR Biomed 2011;24:452-57

33. Hori M, Fukunaga I, Masutani Y, et al. New diffusion metrics for spondylotic myelopathy at an early clinical stage. Eur Radiol 2012;22:1797-802

34. Polman CH, Reingold SC, Edan G, et al. Diagnostic criteria for multiple sclerosis: 2005 revisions to the "McDonald Criteria." Ann Neurol 2005;58:840-46

35. Reese TG, Heid O, Weisskoff RM, et al. Reduction of eddy-currentinduced distortion in diffusion MRI using a twice-refocused spin echo. Magn Reson Med 2003;49:177-82

36. Jones DK, Horsfield MA, Simmons A. Optimal strategies for measuring diffusion in anisotropic systems by magnetic resonance imaging. Magn Reson Med 1999;42:515-25

37. Skare S, Li T, Nordell B, et al. Noise considerations in the determination of diffusion tensor anisotropy. Magn Reson Imaging 2000;18:659-69

38. Tabesh A, Jensen JH, Ardekani BA, et al. Estimation of tensors and tensor-derived measures in diffusional kurtosis imaging. Magn Reson Med 2011;65:823-36

39. Freund P, Wheeler-Kingshott C, Jackson J, et al. Recovery after spinal cord relapse in multiple sclerosis is predicted by radial diffusivity. Mult Scler 2010;16:1193-202

40. Losseff NA, Webb SL, O'Riordan JI, et al. Spinal cord atrophy and disability in multiple sclerosis: a new reproducible and sensitive MRI method with potential to monitor disease progression. Brain 1996;119(pt 3):701-08

41. Mikheev A, Nevsky G, Govindan S, et al. Fully automatic segmentation of the brain from T1-weighted MRI using Bridge Burner algorithm. J Magn Reson Imaging 2008;27:1235-41

42. Rashid W, Davies GR, Chard DT, et al. Increasing cord atrophy in early relapsing-remitting multiple sclerosis: a 3 year study. J Neurol Neurosurg Psychiatr 2006;77:51-55

43. DeLuca GC, Ebers GC, Esiri MM. Axonal loss in multiple sclerosis: a pathological survey of the corticospinal and sensory tracts. Brain 2004;127:1009-18

44. Mottershead JP, Schmierer K, Clemence M, et al. High field MRI correlates of myelin content and axonal density in multiple sclerosis: a post-mortem study of the spinal cord. J Neurol 2003;250:1293-301

45. Bot JC, Blezer EL, Kamphorst W, et al. The spinal cord in multiple sclerosis: relationship of high-spatial-resolution quantitative MR imaging findings to histopathologic results. Radiology 2004;233:531-40

46. Gilmore CP, Donaldson I, Bö L, et al. Regional variations in the extent and pattern of grey matter demyelination in multiple sclerosis: a comparison between the cerebral cortex, cerebellar cortex, deep grey matter nuclei and the spinal cord. J Neurol Neurosurg Psychiatr 2009;80:182-87

47. Jensen JH, Helpern JA, Ramani A, et al. Diffusional kurtosis imaging: the quantification of non-gaussian water diffusion by means of magnetic resonance imaging. Magn Reson Med 2005;53: $1432-40$ 